\title{
Dual Decomposition Methods for Nonlinear Resource Allocation Problems in Telecommunication Networks
}

\author{
Igor Konnov \\ Department of System Analysis \\ and Information Technologies \\ Kazan Federal University \\ Kazan 420008, Russia \\ Email: konn-igor@ya.ru
}

\author{
Aleksey Kashuba \\ LLC "AST Povolzhye" \\ Kazan, 420029, Russia \\ Email: leksser@rambler.ru
}

\author{
Erkki Laitinen \\ Department of Mathematical Sciences \\ University of Oulu \\ 90014 Oulu, Finland \\ Email: erkki.laitinen@oulu.fi
}

\begin{abstract}
We consider problems of optimal resource allocation in zonal telecommunication networks with many users. In the simplest formulation the network manager aims to distribute some homogeneous resource (say bandwidth) among users within one zone. We assume strictly convex charge and convex quadratic fee functions and present combined dual type solution methods. Next, we consider a more general problem for a multizonal wireless communication network with common capacity constraints. We obtain a convex optimization problem involving two kinds of constraints. By using the dual Lagrangian method with respect to the capacity constraint, we suggest to reduce the initial problem to a single-dimensional optimization problem, but calculation of the cost function value leads to independent solution of zonal problems, which coincide with the previous single region problem. Some results of computational experiments confirm the applicability of the new methods.

Index Terms-Resource allocation, wireless networks, multizonal networks, affine price functions, nonlinear charge functions, convex optimization, Lagrangian duality, decomposition.
\end{abstract}

\section{INTRODUCTION}

Due to strong variability and increasing demand of different wireless telecommunication services, fixed allocation rules usually lead to serious congestion effects and inefficient utilization of network resources despite the presence of very powerful processing and transmission devices. Hence, one has to find more flexible allocation mechanisms instead of the fixed allocation ones. These mechanisms are based on proper mathematical models; see e.g. [1]-[3]. In particular, spectrum sharing is now one of the most critical issues in this field and various adaptive mechanisms have been suggested. Treatment of these very complicated systems is often based on a proper decomposition/clustering approach, which can involve zonal, time, frequency and other decomposition procedures for nodes/units; see e.g. [4], [5], [6], [7].

In this work, the first and second authors were supported by the RFBR grant, project No. 16-01-00109a. The first and third authors were supported by by grant No. 297689 from Academy of Finland. Also, the work of the second author is performed within the Government Program of Competitive Growth of Kazan Federal University.
In this paper, we consider a basic problem of optimal allocation of a homogeneous resource in telecommunication networks such that the income received from users payments is maximized and the implementation costs of the network operator are minimized. We first present an optimization formulation for the case where the network manager aims to distribute some homogeneous resource (bandwidth) among users of one region with convex charge and quadratic fee functions. We show that these convex optimization problems can be solved by dual type iterative methods. Namely, we propose to apply the bisection method and the conditional gradient method combined with the solution of the dual problem.

Next, we consider a more general resource allocation problem for a provider of a multi-zonal wireless communication network, which was formulated as a convex optimization problem in [8], [9]. Now, since the price functions of buyers are affine and the price functions of the network provider are monotone, we obtain again a convex optimization problem having capacity and zonal balance constraints. By using the dual Lagrangian method with respect to the capacity constraint, we suggest to reduce the initial problem to a single-dimensional optimization problem, but calculation of the cost function value leads to independent solution of zonal problems, which coincide with the previous single region problem. We present results of computational experiments which confirm the applicability of the new methods.

\section{Single-ZONAL RESOURCE ALLOCATION MODEL}

Following [10], we first consider a single-zonal telecommunication network with one provider. The general problem of a network manager is to find an optimal allocation of a limited homogeneous resource among the users (nodes) in order to maximize the total payment received from the users and to minimize the total network implementation expenses. That is, $x$ is an unknown quantity of the resource offered by the network, with the capacity bounds $x \in[0, b]$, which yields the network expense (cost of implementation) $u(x)$. Similarly, $y_{i}$ is the unknown resource offered to user $i \in I$ and $\varphi_{i}\left(y_{i}\right)$ 
is the fee (incentive) value paid by user $i$ with the capacity bounds $y_{i} \in\left[0, a_{i}\right]$, where $I$ is the index set of users. The network manager problem is then formulated as follows:

$$
\max _{(x, y) \in D} \rightarrow \mu(x, y)=\left\{\sum_{i \in I} \varphi_{i}\left(y_{i}\right)-u(x)\right\},
$$

where $y=\left(y_{i}\right)_{i \in I}$,

$$
D=\left\{(x, y) \mid \sum_{i \in I} y_{i}=x, 0 \leq y_{i} \leq a_{i}, i \in I, 0 \leq x \leq b\right\} \text {. }
$$

Suppose that the set $D$ is non-empty, the function $u(x)$ is strictly convex and differentiable, the functions $\varphi_{i}\left(y_{i}\right)$ are concave and quadratic, i.e.,

$$
\varphi_{i}\left(y_{i}\right)=0.5 \alpha_{i} y_{i}^{2}+\beta_{i} y_{i}+\sigma_{i}, \alpha_{i}<0, i \in I .
$$

Then (1) is a differentiable convex optimization problem, which is treated as a two-side market model. Unlike [10], we do not insist that the function $u(x)$ be also quadratic. This means that the provider price function may be nonlinear in general, which corresponds to a more general model of behavior, but requires special solution methods since those from [10] are not applicable now.

Let $g(x)=u^{\prime}(x)$ and $h_{i}\left(y_{i}\right)=\varphi_{i}^{\prime}\left(y_{i}\right)$. The necessary and sufficient optimality condition for problem (1) is written in the form of the variational inequality: find $(\bar{x}, \bar{y}) \in D$ such that

$$
g(\bar{x})(x-\bar{x})-\sum_{i \in I} h_{i}\left(\bar{y}_{i}\right)\left(y_{i}-\bar{y}_{i}\right) \geq 0, \forall(x, y) \in D .
$$

In this case optimality conditions can be written as

$$
(\bar{x}, \bar{y}) \in D, \exists p^{*}, \quad g(\bar{x}) \begin{cases}\geq p^{*} & \text { if } \bar{x}=0, \\ =p^{*} & \text { if } \bar{x} \in(0, b), \\ \leq p^{*} & \text { if } \bar{x}=b ;\end{cases}
$$

and

$$
h_{i}\left(\bar{y}_{i}\right) \begin{cases}\leq p^{*} & \text { if } \bar{y}_{i}=0, \\ =p^{*} & \text { if } \bar{y}_{i} \in\left(0, a_{i}\right), \quad \text { for } i \in I ; \\ \geq p^{*} & \text { if } \bar{y}_{i}=a_{i},\end{cases}
$$

This is precisely a two-side market model with one trader where all the buyers have affine price functions; see [11], [12]. Therefore, this is the case for the convex optimization problem (1).

Following the dual approach, we write the Lagrange function of problem (1) with the negative sign:

$$
\begin{aligned}
M(x, y, p) & =u(x)-\sum_{i \in I} \varphi_{i}\left(y_{i}\right)-p\left(x-\sum_{i \in I} y_{i}\right) \\
& =(u(x)-p x)-\sum_{i \in I}\left(\varphi_{i}\left(y_{i}\right)-p y_{i}\right) .
\end{aligned}
$$

In order to find a value of the dual cost function

$$
\theta(p)=\min _{x \in[0, b], y \in[0, a]} M(x, y, p),
$$

where $a=\left(a_{i}\right)_{i \in I}$, we have to solve one- dimensional problems:

$$
\min _{0 \leq x_{k} \leq b_{k}} \rightarrow(u(x)-p x),
$$

and

$$
\max _{0 \leq y_{i} \leq a_{i}} \rightarrow\left(0.5 \alpha_{i} y_{i}^{2}+\beta_{i} y_{i}-p y_{i}\right)
$$

for $i \in I$. Solutions of these problems, which are denoted by $x(p)$ and $y_{i}(p), i \in I$, respectively, are defined uniquely.

It follows that the function $\theta(p)$ is concave and differentiable with

$$
\theta^{\prime}(p)=\sum_{i \in I} y_{i}(p)-x(p) .
$$

Besides, the one- dimensional dual problem

$$
\max _{p} \rightarrow \theta(p)
$$

coincides with the simple equation

$$
\theta^{\prime}(p)=0,
$$

where $\theta^{\prime}(p)$ is non-increasing. If $p^{*}$ is a solution of (4), then we can find the solution of the initial problem (1) from (2)-(3) by setting $p=p^{*}$.

Let us describe first the dual bisection algorithm named Algorithm (BS). It is based on the above treatment of the convex optimization problem (1) as a two-side market model.

Set $\gamma^{\prime}=g(0)$ and $\gamma^{\prime \prime}=g(b)$. Then $\gamma^{\prime}<\gamma^{\prime \prime}$. Set also $\delta_{i}^{\prime}=\beta_{i}$ and $\delta_{i}^{\prime \prime}=\alpha_{i} a_{i}+\beta_{i}$ for $i \in I$.

If we define $p^{\prime \prime}=\max _{i \in I} \delta_{i}^{\prime}$ and $p^{\prime}=\gamma^{\prime}$, then the case $p^{\prime \prime} \leq p^{\prime}$ yields immediately the zero solutions in accordance with (2)(3). So we can consider only the non-trivial case where $p^{\prime}<$ $p^{\prime \prime}$. Then by (2)-(3) we must have $\theta^{\prime}\left(p^{\prime}\right)>0$ and $\theta^{\prime}\left(p^{\prime \prime}\right)<0$. These properties enable us to find a solution of (4) by the simple bisection algorithm. Given an accuracy $\varepsilon>0$ and the initial segment $\left[p^{\prime}, p^{\prime \prime}\right]$, we take $\tilde{p}=0.5\left(p^{\prime}+p^{\prime \prime}\right)$, calculate $\theta^{\prime}(\tilde{p})$. Then we set $p^{\prime}=\tilde{p}$ if $\theta^{\prime}(\tilde{p})>0$ and $p^{\prime}=\tilde{p}$ otherwise, until $\left(p^{\prime \prime}-p^{\prime}\right)<\varepsilon$.

Following [13], we can take the conditional gradient method with linear search (CGDM) for problem (1). For simplicity, set $\tilde{\mu}(w)=-\mu(x, y)$.

Take an arbitrary initial point $w^{0} \in D$ and numbers $\alpha \in$ $(0,1), \gamma \in(0,1)$, and $\delta>0$. At the $k$-th iteration, $k=$ $0,1, \ldots$, we have a point $w^{k} \in D$ and calculate $u^{k} \in D$ as a solution of the linear programming problem

$$
\min _{w \in D} \rightarrow\left\langle\tilde{\mu}^{\prime}\left(w^{k}\right), w\right\rangle .
$$

Then we set $p^{k}=u^{k}-w^{k}$. If $\left\|p^{k}\right\| \leq \delta$, stop, we have an approximate solution. Otherwise we find $m$ as the minimal non-negative integer such that

$$
\tilde{\mu}\left(w^{k}+\gamma^{m} p^{k}\right) \leq \tilde{\mu}\left(w^{k}\right)+\alpha \gamma^{m}\left\langle\tilde{\mu}^{\prime}\left(w^{k}\right), p^{k}\right\rangle,
$$

set $\theta_{k}=\gamma^{m}, w^{k+1}=w^{k}+\theta_{k} p^{k}$, and $k=k+1$.

The main idea of this approach is that the basic linear programming problem (5) is written as a two-side market model with one trader and many buyers having fixed prices. Hence, it can be solved in a finite number of iterations by a simple arrangement type procedure; see [14]. 


\section{MULTI-ZONAL NETWORK PROBLEM}

Now we consider a more general model where a telecommunication network is divided into several zones (clusters). The problem of a manager of the whole network is also to find the optimal allocation of a limited homogeneous network resource among the zones in order to maximize the total profit containing the total income received from consumers' fees and negative resource implementation costs; see [8], [9].

Let us use the following notation:

- $n$ is the number of zones;

- $I_{k}$ is the index set of users (currently) located in zone $k$ $(k=1, \ldots, n)$;

- $B$ is the total resource supply (the total bandwidth) for the system (network);

- $x_{k}$ is an unknown quantity of the resource allotted to zone $k$ with the upper bound $b_{k}$ and $f_{k}\left(x_{k}\right)$ is the cost of implementation of this quantity of the resource for zone $k(k=1, \ldots, n)$;

- $y_{i}$ is the resource amount received by user $i$ with the upper bound $a_{i}$ and $\varphi_{i}\left(y_{i}\right)$ is the charge value paid by user $i$ for the resource value $y_{i}$.

The network manager problem is the optimization problem involving capacity and balance constraints:

$$
\max \rightarrow \sum_{k=1}^{n}\left[\sum_{i \in I_{k}} \varphi_{i}\left(y_{i}\right)-f_{k}\left(x_{k}\right)\right],
$$

subject to

$$
\begin{aligned}
& \sum_{k=1}^{n} x_{k} \leq B \\
& \sum_{i \in I_{k}} y_{i}=x_{k}, k=1, \ldots, n \\
& 0 \leq y_{i} \leq a_{i}, \quad i \in I_{k}, 0 \leq x_{k} \leq b_{k}, k=1, \ldots, n .
\end{aligned}
$$

That is, (8) provides the balance for demand and supply in each zone, (9) involves capacity constraints for users and network supply values in each zone, and (7) gives the upper bound for the total resource supply.

In what follows we assume that there exists at least one feasible point satisfying conditions (7)-(9), all the functions $f_{k}\left(x_{k}\right)$ are strictly convex and differentiable and all the functions $\varphi_{i}\left(y_{i}\right)$ are concave and quadratic, i.e.

$$
\begin{gathered}
\varphi_{i}\left(y_{i}\right)=0.5 \alpha_{i} y_{i}^{2}+\beta_{i} y_{i}+\sigma_{i}, \alpha_{i}<0, i \in I_{k}, \\
k=1, \ldots, n .
\end{gathered}
$$

This means that (6)-(10) is a convex optimization problem. However, due to large dimensionality and inexact data one can meet serious drawbacks in solving this problem with usual finite or penalty solution methods. In order to create an efficient method, we have to take into account its separability and apply certain decomposition approach. However, the standard duality approach using the Lagrangian function with respect to all the functional constraints leads to the multi-dimensional dual optimization problem. We will apply another approach, which was suggested in [15]. Let us define the Lagrange function of problem (6)-(9) as follows:

$L(x, y, \lambda)=\sum_{k=1}^{n}\left[\sum_{i \in I_{k}} \varphi_{i}\left(y_{i}\right)-f_{k}\left(x_{k}\right)\right]-\lambda\left(\sum_{k=1}^{n} x_{k}-B\right)$.

We utilize the Lagrangian multiplier $\lambda$ only for the total resource bound. We can now replace problem (6)-(9) with its dual:

$$
\min _{\lambda \geq 0} \rightarrow \psi(\lambda)
$$

where

$$
\begin{array}{r}
\psi(\lambda)=\max _{(x, y) \in W} L(x, y, \lambda)=\lambda B \\
+\max _{(x, y) \in W} \sum_{k=1}^{n}\left[\sum_{i \in I_{k}} \varphi_{i}\left(y_{i}\right)-f_{k}\left(x_{k}\right)-\lambda x_{k}\right], \\
W=\left\{(x, y) \mid \begin{array}{l}
\sum_{i \in I_{k}} y_{i}=x_{k}, 0 \leq y_{i} \leq a_{i}, i \in I_{k}, \\
0 \leq x_{k} \leq b_{k}, k=1, \ldots, n
\end{array}\right\} .
\end{array}
$$

By duality (see e.g. [16], [17]), problems (6)-(9) and (11) have the same optimal value. But the solution of (11) can be found by one of well-known single-dimensional optimization algorithms; see e.g. [17]. In order to calculate the value of $\psi(\lambda)$ we have to solve the inner problem:

$$
\max \rightarrow \sum_{k=1}^{n}\left[\sum_{i \in I_{k}} \varphi_{i}\left(y_{i}\right)-f_{k}\left(x_{k}\right)-\lambda x_{k}\right],
$$

subject to

$$
\begin{aligned}
& \sum_{i \in I_{k}} y_{i}=x_{k}, 0 \leq y_{i} \leq a_{i}, \quad i \in I_{k}, \\
& 0 \leq x_{k} \leq b_{k}, k=1, \ldots, n .
\end{aligned}
$$

Obviously, this problem decomposes into $n$ independent zonal optimization problems

$$
\max \rightarrow\left[\sum_{i \in I_{k}} \varphi_{i}\left(y_{i}\right)-f_{k}\left(x_{k}\right)-\lambda x_{k}\right],
$$

subject to

$$
\begin{aligned}
& \sum_{i \in I_{k}} y_{i}=x_{k}, 0 \leq y_{i} \leq a_{i}, \quad i \in I_{k}, \\
& 0 \leq x_{k} \leq b_{k}
\end{aligned}
$$

for $k=1, \ldots, n$. Each $k$-th independent zonal problem (12) clearly coincides with problem (1) where

$$
\begin{aligned}
& \varphi_{i}\left(y_{i}\right)=0.5 \alpha_{i} y_{i}^{2}+\beta_{i} y_{i}+\sigma_{i}, i \in I_{k}, \\
& u(x)=f_{k}\left(x_{k}\right)+\lambda x_{k} .
\end{aligned}
$$

Therefore, we can find its solution by the algorithms described in Section II. 
TABLE I

RESULTS OF TESTING WITH $J=510, n=70$

\begin{tabular}{|c|l|l|l|}
\hline$\varepsilon_{\lambda}$ & $T_{\varepsilon}:(\mathrm{CGDM} 0)$ & $T_{\varepsilon}:(\mathrm{CGDMB})$ & $T_{\varepsilon}:$ Algorithm (BS) \\
\hline $10^{-1}$ & 0.0275 & 2.3406 & 0.0013 \\
\hline $10^{-2}$ & 0.0322 & 2.9034 & 0.0019 \\
\hline $10^{-3}$ & 0.0413 & 3.3624 & 0.0025 \\
\hline $10^{-4}$ & 0.0463 & 3.8750 & 0.0019 \\
\hline
\end{tabular}

TABLE II

RESULTS OF TESTING WITH $n=70, \varepsilon=10^{-2}$

\begin{tabular}{|c|l|l|l|}
\hline$J$ & $T_{\varepsilon}:($ CGDM0) & $T_{\varepsilon}:($ CGDMB) & $T_{\varepsilon}:$ Algorithm (BS) \\
\hline 210 & 0.0078 & 0.2344 & 0.0003 \\
\hline 310 & 0.0159 & 0.7248 & 0.0003 \\
\hline 410 & 0.0248 & 1.7156 & 0.0006 \\
\hline 510 & 0.0322 & 2.9034 & 0.0019 \\
\hline 610 & 0.0457 & 4.2282 & 0.0015 \\
\hline 710 & 0.0603 & 5.6814 & 0.0025 \\
\hline 810 & 0.0741 & 7.4378 & 0.0041 \\
\hline 910 & 0.0891 & 9.3688 & 0.0035 \\
\hline 1010 & 0.1071 & 11.5718 & 0.0053 \\
\hline
\end{tabular}

\section{NUMERICAL EXPERIMENTS}

In order to evaluate efficiency of the new method we made several series of computational experiments. The programs on the methods were coded in $\mathrm{C}++$ with a PC with the following facilities: Intel(R) Core(TM) i7-4500, CPU $1.80 \mathrm{GHz}, \mathrm{RAM}$ $6 \mathrm{~Gb}$.

The initial intervals for choosing the dual variable $\lambda$ were taken as $[0,1000]$. Values of $b_{k}$ were chosen by trigonometric functions in [1,51], values of $a_{i}$ were chosen by trigonometric functions in $[1,2]$. Value $B$ were taken equal 1000. Values $\gamma$ and $\alpha$ in (CGDM) was chosen to be 0.7 and 0.4 , respectively. The number of zones was varied from 5 to 1005 , the number of users was varied from 210 to 10010 . Users were distributed in zones either uniformly or according to the normal distribution. We took the cost functions $f_{k}\left(x_{k}\right)=\tau_{k} \exp \left(\eta_{k} x_{k}\right)$. The coefficients of the functions $f_{k}\left(x_{k}\right)$ and $\varphi_{i}\left(y_{i}\right)$ from (10) were taken as

$$
\tau_{k}=|\cos (2 k+2)|+1, \eta_{k}=|\cos (k+1)|+3,
$$

and

$$
\alpha_{i}=-3|\cos (2 i+1)|-3, \beta_{i}=|\sin (i+2)|-1 .
$$

For all the methods of finding a solution of problem (6)-(9) the accuracy of the upper dual problem solution were varied from $10^{-1}$ to $10^{-4}$. The accuracy of the lower level problem solution was fixed and equal to $10^{-2}$. For each set of the parameters we made 50 tests. Let $J$ denote the total number of users, $T_{\varepsilon}$ the total processor time in seconds. We named by (CGDM0) a dual Lagrangian method with conditional gradient method with linear search with initial point $w^{0}=0$ and (CGDMB) a dual Lagrangian method with conditional gradient method with linear search with initial point $w^{0}$ equaled upper boundary of $\tilde{W}$. The results of computations are given in Tables I-IV.
TABLE III

RESUlTS OF TESTING WITH $J=510, \varepsilon=10^{-2}$

\begin{tabular}{|c|l|l|l|}
\hline$n$ & $T_{\varepsilon}:(\mathrm{CGDM})$ & $T_{\varepsilon}:(\mathrm{CGDMB})$ & $T_{\varepsilon}:$ Algorithm $(\mathrm{BS})$ \\
\hline 5 & 0.0284 & 3.3936 & 0.0015 \\
\hline 15 & 0.0290 & 3.3470 & 0.0009 \\
\hline 25 & 0.0290 & 3.2906 & 0.0009 \\
\hline 35 & 0.0284 & 3,1690 & 0.0012 \\
\hline 45 & 0.0297 & 3,0910 & 0.0012 \\
\hline 55 & 0.0307 & 2.9750 & 0.0028 \\
\hline 65 & 0.0305 & 2,9220 & 0.0012 \\
\hline 75 & 0.0325 & 2.8588 & 0.0015 \\
\hline 85 & 0.0332 & 2.8376 & 0.0028 \\
\hline 95 & 0.0331 & 2.8068 & 0.0022 \\
\hline 105 & 0.0334 & 2,7940 & 0.0027 \\
\hline
\end{tabular}

TABLE IV

RESULTS OF TESTING WITH $n=70, \varepsilon=10^{-2}$

\begin{tabular}{|c|l|l|l|}
\hline$J$ & $T_{\varepsilon}:($ CGDM0) & $T_{\varepsilon}:($ CGDMB) & $T_{\varepsilon}:$ Algorithm (BS) \\
\hline 1010 & 0.1071 & 11.5718 & 0.0053 \\
\hline 2010 & 0.4308 & 22.6720 & 0.0313 \\
\hline 3010 & 0.9846 & 62.5000 & 0.1161 \\
\hline 4010 & 1.7436 & 140.1100 & 0.2141 \\
\hline 5010 & 2.7354 & 239.3160 & 0.3428 \\
\hline 6010 & 3.8752 & 354.0210 & 0.5000 \\
\hline 7010 & 5.3212 & 496.8820 & 0.6888 \\
\hline 8010 & 6.9454 & 671.1140 & 0.9225 \\
\hline 9010 & 8.8922 & 872.3650 & 1.2275 \\
\hline 10010 & 11.0104 & 1129.3900 & 1.6335 \\
\hline
\end{tabular}

As we can see from the results in the tables, in all the cases the suggested methods were capable to find a solution. The processor time expenses given in the tables show that utilization of Algorithm (BS) for inner optimization problems give better performance, especially on large problems (see Table IV).

\section{CONCLuSions}

We considered a basic problem of optimal allocation of a homogeneous resource in telecommunication networks. We presented combined dual type solution methods for the case where the network manager aims to distribute some homogeneous resource (bandwidth) among users of one region with convex charge and quadratic fee functions. Next, we considered a more general problem for a provider of a wireless communication network divided into several zones. By using the dual Lagrangian method, we transformed this problem into a two-level problem with the single-dimensional optimization problem at the upper level and the set of independent zonal problems at the lower level. The results of computational experiments confirmed the applicability of the new methods.

\section{REFERENCES}

[1] Courcoubetis, C., Weber, R.: Pricing Communication Networks: Economics, Technology and Modelling. John Wiley \& Sons, Chichester (2003)

[2] Stańczak, S., Wiczanowski, M., Boche, H.: Resource Allocation in Wireless Networks. Theory and Algorithms. Springer, Berlin (2006)

[3] Wyglinski, A.M., Nekovee, M., Hou, Y.T.: (eds.) Cognitive Radio Communications and Networks: Principles and Practice. Elsevier, Amsterdam (2010) 
[4] Chen, Y., Liestman, A.L.: A zonal algorithm for clustering ad hoc networks. Int. J. Found. of Computer Sci., vol.14, pp.305-322 (2003)

[5] Rohloff, K., Ye, J., Loyall, J., Schantz, R.: A hierarchical control system for dynamic resource management. Proc. of the 12th IEEE Real-Time and Embed. Technol. and Appl. Symposium (RTAS 2006). Work in Progress Symposium, San Jose, (2006)

[6] Nordin, R.: Interference-aware subcarrier allocation in a correlated MIMO downlink transmission. WSEAS Trans. on Comm., vol. 11, pp. 158-169 (2012)

[7] Huang, X., Leng, S., Wu, F., Mao, Y.: Energy-efficient and QoSware resource allocation for relay-enhanced OFDMA wireless cellular networks. WSEAS Trans. on Comm., vol. 13, pp. 394-401 (2014)

[8] Konnov, I.V., Kashina, O.A., Laitinen, E.: Optimisation problems for control of distributed resources. Int. J. Model., Ident. and Contr., vol.14, pp.65-72 (2011)

[9] Konnov, I.V., Kashina, O.A., Laitinen, E.: Two-level decomposition method for resource allocation in telecommunication network. Int. J. Dig. Inf. Wirel. Comm., vol.2, pp.150-155 (2012)

[10] Konnov, I.V., Kashuba, A.Yu.: Decomposition method for zonal resource allocation problems in telecommunication networks. IOP Conf. Series: Materials Science and Engineering, vol. 158, Proc. of the 11th International Conference on "Mesh Methods for Boundary-value Problems and Applications", Art. No. 012054. 7 pp. (2016)

[11] Konnov, I.V.: On modeling of auction type markets. Issled. Inform., vol.10, pp.73-76 (2006), Available at SSRN: http://ssrn.com/abstract=2482282

[12] Konnov, I.V.: An alternative economic equilibrium model with different implementation mechanisms. Adv. Model. Optim., vol. 17, pp.245-265 (2015)

[13] Konnov, I.V., Kashuba, A.Yu., Laitinen, E.: Application of the conditional gradient method to resource allocation in wireless networks. Lobachevskii J. Mathem., vol.37, pp.626-635 (2016)

[14] Konnov, I.V. Modelling of auction type markets. Universita degli Studi di Bergamo, DMSIA Report No. 7. Bergamo, Italy. 28 pp. (2007)

[15] Konnov, I.V., Kashuba, A.Yu., Laitinen, E.: A simple dual method for optimal allocation of total network resources. Recent Advances in Mathematics. Proceedings of the International Conference "PMAMCM 2015”. Ed. by I.J. Rudas. Zakynthos, pp.19-21 (2015)

[16] Polyak, B.T.: Introduction to Optimization. Nauka, Moscow (1983) [Engl. transl. in Optimization Software, New York, 1987]

[17] Konnov, I.V.: Nonlinear Optimization and Variational Inequalities. Kazan Univ. Press, Kazan (2013) 